\title{
CNT EUV pellicle: moving towards a full-size solution
}

\section{Marina Y. Timmermans, Ivan Pollentier, Jae Uk Lee, Johan Meersschaut, Olivier Richard, et al.}

Marina Y. Timmermans, Ivan Pollentier, Jae Uk Lee, Johan Meersschaut, Olivier Richard, Christoph Adelmann, Cedric Huyghebaert, Emily E.

Gallagher, "CNT EUV pellicle: moving towards a full-size solution," Proc. SPIE 10451, Photomask Technology, 104510P (16 October 2017); doi: 10.1117/12.2280632

SPIE Event: SPIE Photomask Technology and EUV Lithography, 2017, Monterey, California, United States 


\title{
CNT EUV pellicle: moving towards a full-size solution
}

\author{
Marina Y. Timmermans*, Ivan Pollentier, Jae Uk Lee, Johan Meersschaut, Olivier Richard, \\ Christoph Adelmann, Cedric Huyghebaert and Emily E. Gallagher* \\ imec, Kapeldreef 75, B3001 Leuven (Belgium)
}

\begin{abstract}
Development of a pellicle membrane to protect the reticle from particles for EUV source powers beyond $250 \mathrm{~W}$ is a subject of intensive research and is in great demand to support high volume manufacturing with EUV lithography. Identifying a membrane with high EUV transmission, mechanical integrity, thermal stability and chemical resistance within the scanner environment is extremely challenging; yet these properties are required to realize next-generation EUV pellicle solutions. This paper proposes free-standing carbon nanotube (CNT) film as an alternative next generation core pellicle material. Most of the desired pellicle characteristics can be achieved by tuning the properties of freestanding CNT films. We demonstrate that free-standing CNT films possess very high EUV transmission (up to 99\%) and good transmission uniformity ( $0.4 \%$ half range), mechanical stability (maximum deflection $\sim 0.08 \mathrm{~mm}$ at $2 \mathrm{~Pa}$ ), thermal stability (no change under greater than $500 \mathrm{~W}$ equivalent EUV exposure in vacuum without hydrogen radicals) and scalability to a full pellicle size. Other important CNT membrane properties are presented and are favourable for the pellicle application: low EUV scattering, low EUV reflectivity and high transmission under DUV. The ability of the CNT film to stop particles is analysed. The only known failure of the CNT membrane is instability to hydrogen radical/ion environment within the current reticle chamber of the scanner. If changing that environment to limit hydrogen radicals near the pellicle surface is not an option, there is a need to coat the CNT structures for protection. The challenges and considerations for coating the free-standing CNT membranes are discussed.
\end{abstract}

Keywords: EUV pellicle, carbon nanotubes, free-standing film, EUV transmission

\section{INTRODUCTION}

EUV pellicles have been in development for over a decade for use during EUV lithography. A pellicle is a very thin membrane suspended above the patterned side of a photomask to keep any fall-on particles out of the focal plane for imaging. Since all materials absorb strongly at the $13.5 \mathrm{~nm}$ EUV exposure wavelength, identifying an EUV pellicle solution is difficult. The fundamental challenge is to fabricate a free-standing membrane that is thin enough to limit the imaging impact, while robust enough to survive handling. During the early exploratory phases of a lithographic generation, pellicles are optional because defect-free wafers are not essential for test designs. Now that high volume manufacturing using EUV lithography is approaching quickly, the need for an EUV pellicle is imminent ${ }^{1}$. ASML has developed a polysilicon based pellicle that is appropriate for use below about $200 \mathrm{~W}$ exposure power but tends to loose strength at higher powers ${ }^{2}$. A full-size membrane that can survive exposure at the higher power levels associated with high volume has yet to be identified.

EUV pellicle membranes based on SiN, graphite, ceramics and other materials have been proposed as an alternative to polysilicon ${ }^{3,45,6}$. Each material is capable of meeting some of the specifications, but none has achieved all. There are so many requirements, that it is important to develop a systematic approach to screening. The first test is EUV transmission. A path towards achieving above $90 \%$ transmission at reasonable material strength is the primary development target. While radiating heat (emissivity) and resisting the hydrogen radicals/ions in the scanner environment are also critically important, both properties can be modified with a coating layer. Then, there are more subtle requirements: transmission uniformity, low EUV reflectivity, low EUV scattering, and low deflection under pressure gradients. Finally, there are qualities that will provide advantages during use in manufacturing such as high transmission at $193 \mathrm{~nm}$ to enable through-pellicle DUV inspection. Of course, any pellicle candidate film must be fabricated to full size to protect the entire printable field of the photomask.

\footnotetext{
*marina.timmermans@imec.be; emily.gallagher@imec.be
} 
In this paper, we explore the properties of EUV pellicle membranes comprised of carbon nanotubes (CNTs). The pellicle can be considered to consist of a core material that provides strength and low EUV transmission combined with coatings that can enhance lifetime as well as emissivity in various ways. CNTs are recognized as an alternative material to replace silicon for next generation microelectronic applications. Analogously, we show that the CNT material is a promising candidate for the future EUV pellicle core material solution, showing advantages over the existing silicon-based material for EUV source powers beyond $250 \mathrm{~W}$. We also highlight the existing challenge of the incompatibility of the CNT material to the current scanner environment. A path towards coating the CNT membrane to protect it from hydrogen radicals/ions in the scanner is introduced.

\section{CORE PELLICLE: CNT MEMBRANE}

\subsection{Fabrication of free-standing CNT membrane}

CNTs are hollow, seamless nano-cylinders of rolled-up graphene sheets. CNTs can be comprised of either a single graphene layer, known as single-walled CNTs (SWCNTs), or made of several concentric cylindrical graphene shells, known as multi-walled CNTs (MWCNTs). Remarkable electrical, mechanical and optical properties of these nano-sized cylindrical structures made CNTs one of the most exciting discoveries in materials science ${ }^{7}$. For example, CNTs were reported to be around 100 times stronger than $s \mathrm{se}^{8}$ and possess thermal conductivity comparable to diamond ${ }^{9}$. Because of their unique properties, CNTs have already found their way to commercially available products like sports equipment and automotive components, water filtration systems, batteries and transparent conductors for touch panel displays ${ }^{10}$. Market opportunities continue to expand and the current CNT production capacity exceeds several thousand tons per year ${ }^{10}$.

Depending on the method of production, the length of CNTs can vary from less than $100 \mathrm{~nm}$ up to several centimeters ${ }^{11}$. The diameter of individual carbon cylinders can be varied from 0.7 to $2 \mathrm{~nm}$ for SWCNTs and from 5 to $30 \mathrm{~nm}$ for MWCNTs. Due to their high surface energy, individual CNTs tend to agglomerate forming bundles that are held together by van der Waals forces. Such CNT bundles can be tens of nanometers in diameter and tens of micrometers in length.

CNTs can be assembled into free-standing films, with a film morphology that is highly dependent on the fabrication process. Thus films of randomly oriented CNTs can be prepared by vacuum filtration processes where CNTs are either grown by the floating catalyst (aerosol) chemical vapor deposition (CVD) synthesis method ${ }^{12}$ or dispersed in a solution ${ }^{13,14}$. Pulling CNT sheets from vertically-oriented CNT arrays (forests) grown by fixed catalyst CVD method results in highly aligned CNT films ${ }^{15}$. CNTs produced with a dry-process provide a clear path towards scalable production of free-standing CNT films. Free-standing CNT films are placed on top of the support frame for testing and use as a pellicle membrane, as shown in Figure 1.

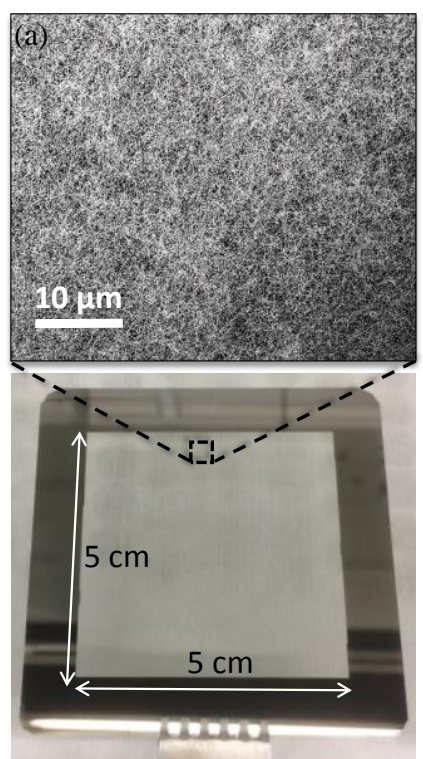

Figure 1: Free-standing CNT films on a support frame $\left(5 \times 5 \mathrm{~cm}^{2}\right)$ :

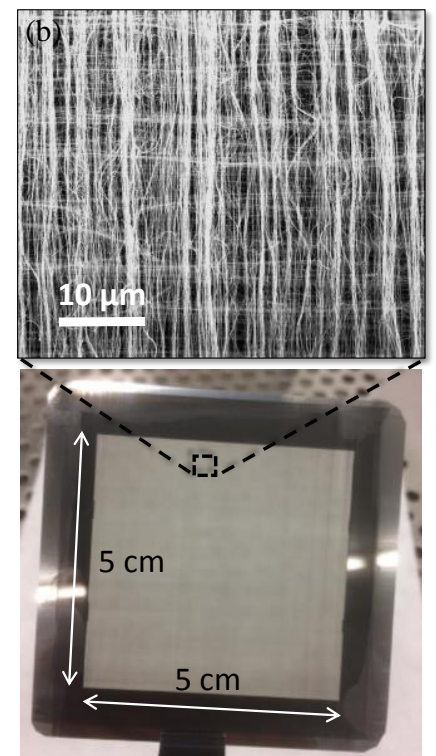

(a) random SWCNTs and (b) aligned MWCNTs. 


\subsection{EUV transmission and uniformity}

EUV transmission is a vital performance parameter for the pellicle material; the development target is greater than $90 \%$ and transmission uniformity of $0.4 \%$ half range ${ }^{16}$. The thickness of free-standing CNT films can be controlled by tuning the CNT density, consequently EUV transmission up to $99 \%$ can be achieved ${ }^{17}$.

EUV uniformity measurements were performed at EUV Tech, Martinez (US) on a $5 \times 5 \mathrm{~cm}^{2} \mathrm{CNT}$ membrane. The membrane survived the transcontinental shipping to be measured there and back. The EUV pellicle transmission tool has a wavelength precision at the $13.5 \mathrm{~nm}$ wavelength of less than $0.01 \mathrm{~nm}$ and the sample was measured at six degrees from normal to match the scanner illumination condition. The measurement spot size was less than $1 \mathrm{x} 1 \mathrm{~mm}^{2}$. The accuracy of the transmission measurements was tested relative to the Advanced Light Source at Berkeley with strong agreement. On our CNT sample, a mean of $96.4 \%$ EUV transmission with a half-range of $0.4 \%$ was achieved. The resulting map is shown in Figure 2. The two local transmission minimum of $96 \%$ are believed to be the consequence of fall-on particles due to handling, but even with that the target performance is achieved.

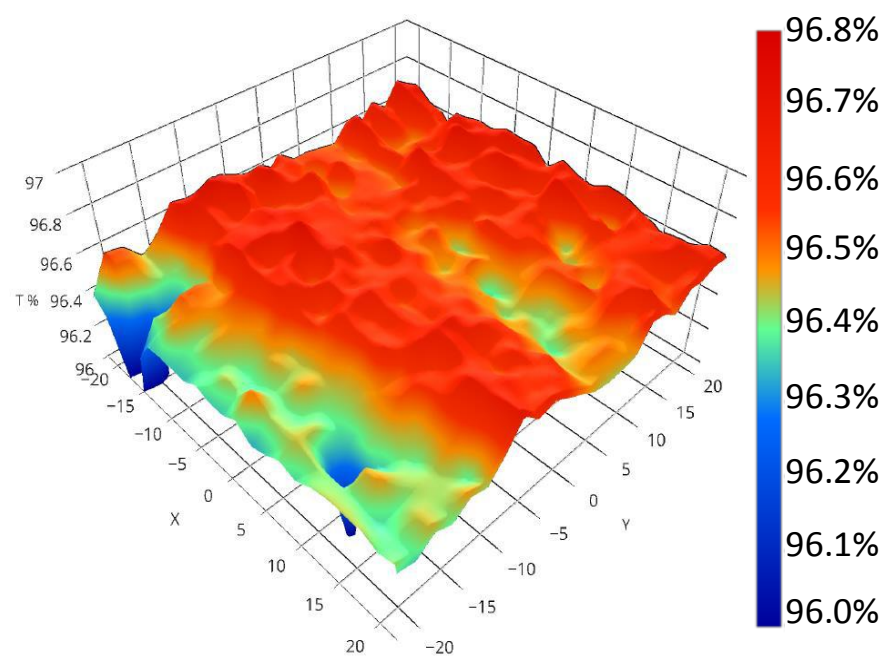

Figure 2: EUV Transmission uniformity measurements on a $5 \times 5 \mathrm{~cm}^{2}$ CNT membrane, measured with the EUV Tech transmission tool. A mean of $96.4 \%$ EUV transmission with a half-range of $0.4 \%$ was achieved. The resulting map is shown in Figure 2. The two local transmission minimum of $96 \%$ are believed to be the consequence of fall-on particles due to handling, but even with that the target performance is achieved.

\subsection{Mechanical properties}

Mechanical stability during shipping and handling in and out of the scanner environment is an important parameter of the pellicle membrane, and an area where free-standing CNT membranes show a significant advantage over conventional solid film approaches. A CNT membrane is a network of CNTs that is porous and therefore allows gas to flow, reducing the pressure difference across the pellicle and minimizing deflection ${ }^{17}$. The physical limitations in the EUV scanner limit the deflection of a full sized pellicle to a maximum of $0.5 \mathrm{~mm}$ at a differential pressure of $<2 \mathrm{~Pa}^{16}$. We have measured the deflection of free-standing CNT films $\left(10 \times 10 \mathrm{~mm}^{2}\right.$ ) with different densities (and hence EUV transmission) with a bulge tester as shown in Figure 3. Thin film SiN membranes exhibit a significant thicknessdependent deflection leading to membrane breakage at the points shown in Figure 3. Thinning the SiN membrane from $100 \mathrm{~nm}$ to $30 \mathrm{~nm}$ increases EUV transmission, but the membrane inevitably suffers from larger deflection (see Figure 3). In contrast to the continuous membrane behaviour, the maximum deflection of the CNT membrane remains fairly constant, at $\sim 0.08 \mathrm{~mm}$ for EUV transmission values of $>65 \%$ without a clear thickness dependence. This constitutes a strong advantage of permeable CNT membrane over traditional thin film pellicle solutions ${ }^{17}$. 


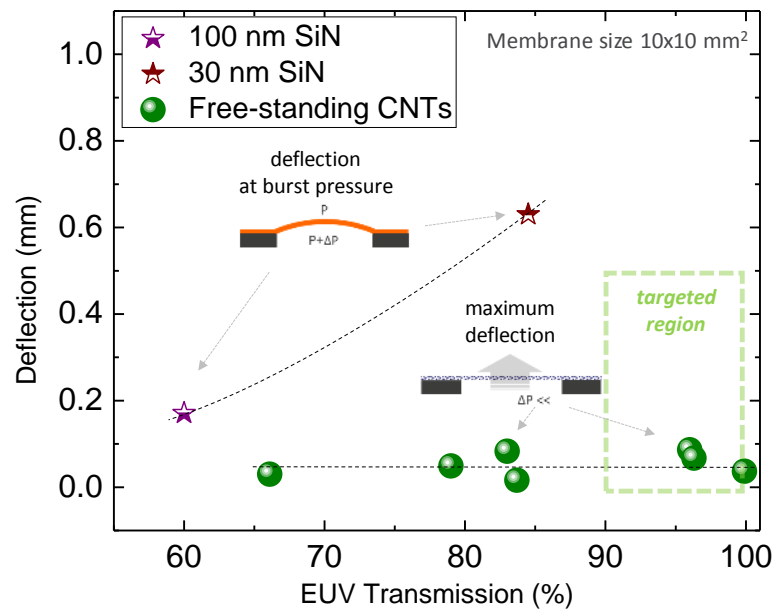

Figure 3: Experimental results of deflection as a function of EUV transmission for solid SiN films (deflection at burst pressure) and free-standing CNT films (maximum deflection). The dashed box indicates the targeted operating region that describes films with high EUV transmission and low defection.

\subsection{Defect mitigation}

Even though the permeability of the CNT membrane gives advantages in dynamic pressure situations, it also presents a risk of particles passing through the gaps in the CNT network, while the primary goal for the pellicle is to avoid that. In contrast to the conventional solid film-based pellicles, this risk needs to be assessed. Initial studies are based on image analysis of SEM pictures of the CNT membrane as is illustrated in Figure 4. The analysis demonstrated here was performed using a CNT membrane with $99 \%$ EUV transmission.

In Figure 4 (a) a typical SEM image is shown at a magnification that is low enough to capture sufficient CNT membrane area to be statistically representative for the membrane, but high enough that the smallest visible gaps are comparable to the image pixel size. After conversion of the grey-scale images to black-and-white using a threshold algorithm, two kinds of image processing are performed.

First, a histogram analysis of the gap area is created. The area of all the black pixels in the picture is calculated, and converted into an equivalent diameter. Note, that is a pessimistic approximation, since the gaps are not circular, nor are they two-dimensional. In reality, a particle would have a more constrained path through a gap. The result of the calculation of the gaps for one example image is shown in Figure 4 (c). Here a normalization is applied to give an estimated gap count over a $1 \mathrm{~mm}^{2}$ area, closer to the real pellicle dimension. It shows that most of the gaps are very small ( 5-10 nm, which is the pixel size), and that the number of larger gaps decreases exponentially. For this CNT geometry, it was found that the maximum gap size was typically below $300 \mathrm{~nm}$, and typically only one such gap is present over the sampled membrane area.

Although the gap size histogram provides insight into the membrane's capacity for stopping large particles, it does not quantify the risk of a smaller particle (e.g. $100 \mathrm{~nm}$ or smaller) to pass through the CNT membrane. For this assessment, a second level of image analysis is done and is illustrated in Figure 4 (b). Each pixel of the image is considered relative to the maximum particle diameter that could pass through that pixel location. When the pixel is white, no particles can pass through. For black pixels, the location of the pixel is important: a pixel located at the edge of a gap can only permit very small particles to pass. Only at the center of a gap can larger particles pass. After mapping all pixels in an image, the histogram of pass-particles can be normalized to the total number of pixels of the sampled area, giving the probability that a certain particle will pass through that small pellicle region. This result is shown in Figure 4 (d). It shows that even for small particles $(30-60 \mathrm{~nm})$ the probability of passing is on the order of a few percent. For larger particles, the probability decreases exponentially. Note again that the obtained result can be considered as a worst-case estimate, since SEMs are not perfect representations of the CNT network: some gap areas could still contain CNTs at very low brightness. Also, the SEM is analyzed perpendicular to the membrane plane, whereas the particle trajectory would be significantly larger for particles incident at shallow angles. We believe that experimental verification to quantify the ability of the CNT pellicle to mitigate defects is important and is currently ongoing. 

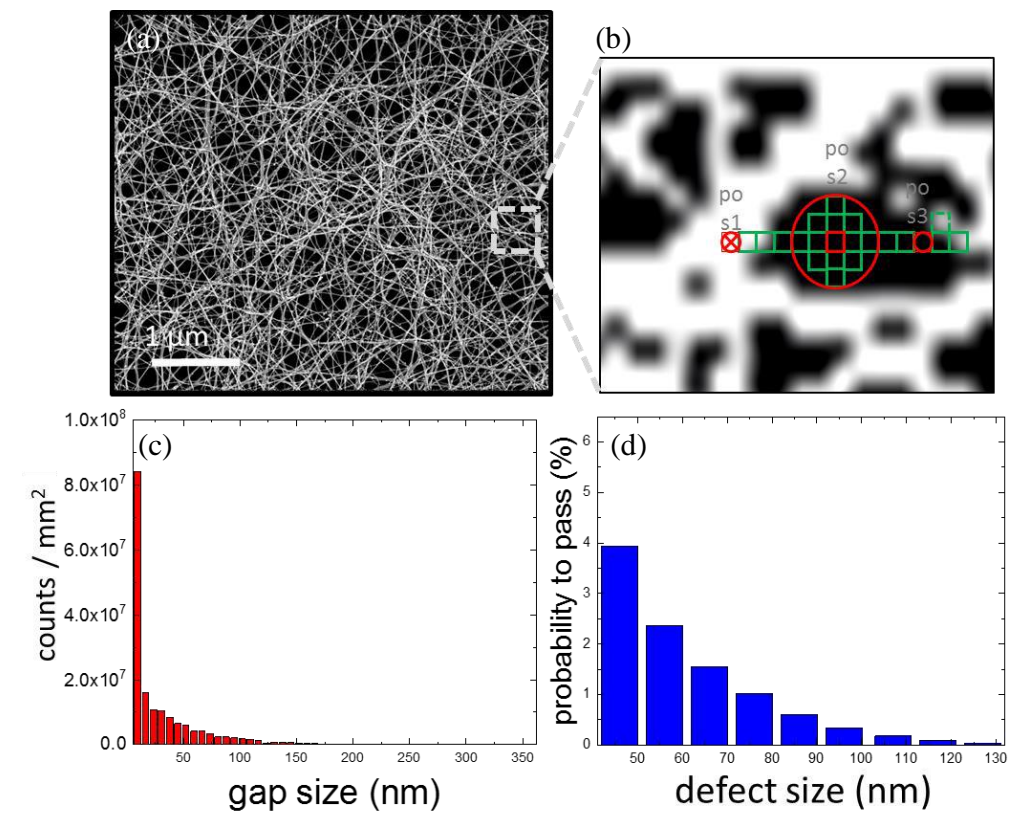

Figure 4: (a) Typical SEM image of a CNT membrane. (b) Processed SEM image for the probability to pass analysis; the grey-scale SEM image is converted to black-and-white by threshold algorithm. (c) Size distribution of gaps within the CNT membrane extrapolated to a $1 \mathrm{~mm}^{2}$ area. (d) Calculated probability of particles passing through the gaps of the CNT membrane with respect to their size. Note, that different image saturation approaches result in differences in the calculated CNT coverage, however variation of the image saturation does not change the calculated probability of particles passage significantly.

\subsection{Thermal stability}

The pellicle membrane's ability to release heat is an essential thermal property at higher EUV exposure powers. In the vacuum environment of the scanner, radiation (emissivity) is the primary transfer mechanism for heat to move from the pellicle material to the surroundings. In our earlier work we discussed the relationship between emissivity and transmission of the pellicle that defines its thermo-optical properties ${ }^{17}$. We have shown that for a material with high EUV transmission, lower emissivity should be sufficient to maintain moderate maximum temperatures ${ }^{17}$. CNT membranes with EUV transmission of $96 \%$ and above show an average emissivity of $\sim 0.02$ which is comparable to solid thin film solutions of $\sim 30 \mathrm{~nm}$ (see Figure 5). Contrary to the case of solid thin films, such a small portion of incoming light is absorbed by the EUV CNT core so its temperature is not expected to rise above $\sim 230^{\circ} \mathrm{C}$. The introduction of active cooling in the scanner ${ }^{18}$ will be able to generate interstitial flow of gas across the CNT membrane, enhancing convection of the pellicle relative to common thin film materials. This superior heat dissipation of porous CNT films has already been studied, demonstrating improved convection because of air flowing across the gaps in the film which allows CNTs at different locations to exchange heat with the surroundings ${ }^{19,20}$.
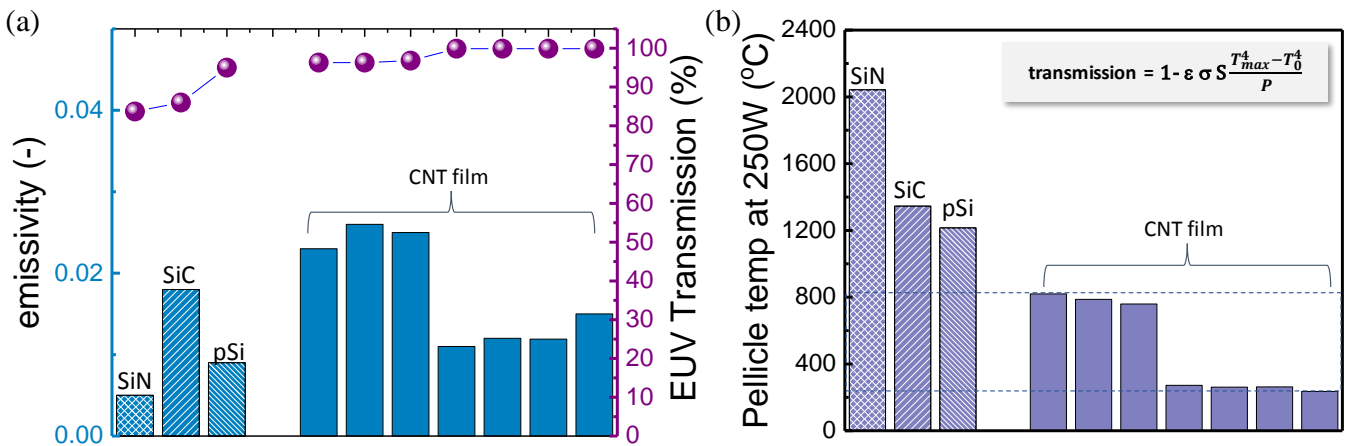

Figure 5: (a) Emissivity and EUV transmission of CNT core material with respect to reference solid films of $30 \mathrm{~nm}$. (b) Calculated expected maximum pellicle temperature at $250 \mathrm{~W}$ source power. 
Individual CNTs are known to be thermally stable up to around $2800^{\circ} \mathrm{C}$ in vacuum or inert atmosphere ${ }^{21}$. CNTs assembled into free-standing films have not been studied with respect to the influence of heating on the film properties, and in particular its EUV transmission. Here, for the first time, we show the thermal stability of free-standing CNT membranes when exposed above $500 \mathrm{~W}$ source power equivalent in vacuum without hydrogen (see table 1).

These heat load tests on the free-standing CNT membrane were performed at PTB metrology institute ${ }^{22}$. The exposure beamline at PTB uses an elliptical mirror at $10^{\circ}$ angle of grazing incidence to focus the radiation of one bending magnet of the BESSY II synchrotron facility. Since the energy of the stored beam cannot be changed, the distance from the focus is varied to change spot size and its power density instead. There are two nominal distances, and hot and cold spots in the beam profile at each position. Thus, it is possible to tune detector distances and the entrance aperture selection to irradiate the pellicle membranes at different power levels to simulate different EUV source powers.

The CNT sample was tested at four different power levels for 30 minutes at each location. A movie was taken during exposure so that signs of failure can be reviewed. For reference, $250 \mathrm{~W}$ exposure corresponds to about $5 \mathrm{~W} / \mathrm{cm}^{2}$ on the pellicle. The conditions used are summarized in the table below. No visible change to the CNT membrane was detected after all the exposure tests. EUV transmission measurements before and after the exposure also showed no change to the CNT membrane.

Table 1. The four EUV power conditions used for core CNT lifetime tests at room temperature without hydrogen.

\begin{tabular}{|l|l|l|l|}
\hline Condition & $\begin{array}{l}\text { Power density } \\
\left(\mathbf{W} / \mathbf{c m}^{2}\right)\end{array}$ & $\begin{array}{l}\text { Source power } \\
\text { equivalent }(\mathbf{W})\end{array}$ & PTB beam conditions \\
\hline 1 & 0.8 & 40 & cold spot at 334 mm focus distance \\
\hline 2 & 2.5 & 125 & $\begin{array}{l}\text { hot spot at } 334 \mathrm{~mm} \text { focus distance or } \\
\text { cold spot at } 161 \mathrm{~mm} \text { focus distance }\end{array}$ \\
\hline 3 & 3.5 & 175 & hot cold spot at 161 mm focus distance \\
\hline 4 & 30 & 1500 & $\begin{array}{l}\text { peak of hot spot at } 161 \mathrm{~mm} \text { focus } \\
\text { distance }\end{array}$ \\
\hline
\end{tabular}

\subsection{Tuning CNT membrane properties}

A unique advantage of the CNT membrane when compared to conventional solid thin films is the flexibility it provides for tuning the intrinsic membrane properties like EUV transmission and emissivity. Figure 6 shows experimental evidence of the ability to alter EUV transmission and emissivity of the CNT membrane by changing the CNT density, diameter of the CNT bundle (amount of CNTs per bundle) and the diameter of individual CNTs that comprise the network.

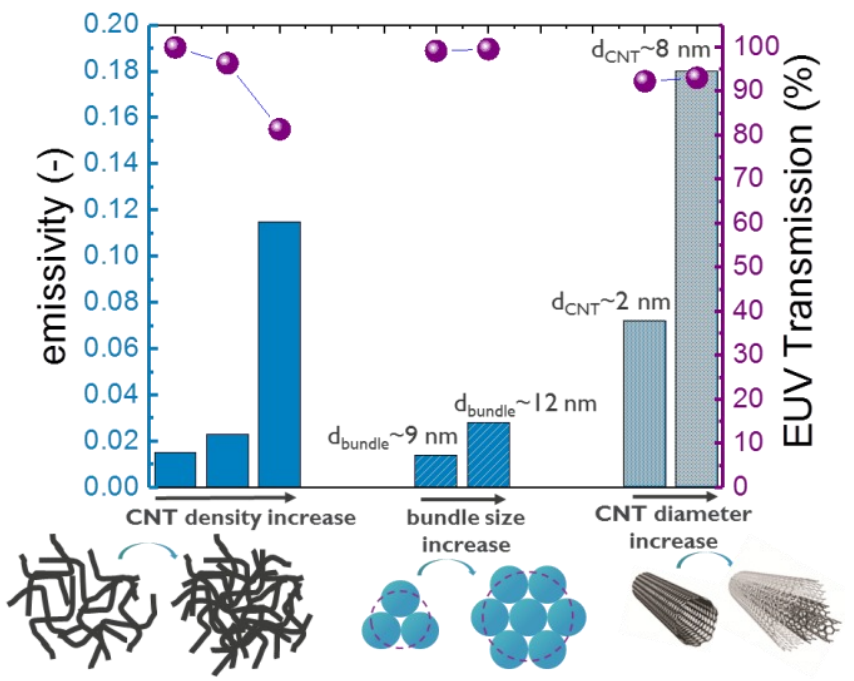

Figure 6: Experimental results demonstrating tunability of the CNT membrane properties, i.e. varying the emissivity and EUV transmission of the CNT membrane by altering the CNT density, bundle diameter (amount of CNTs per bundle) and the diameter of individual CNTs. 
Increasing the number of CNTs within the free-standing CNT film, i.e. CNT density, increases the emissivity at the cost of decreasing the EUV transmission. One goal of tuning the CNT film density is to increase the EUV transmission to above $90 \%$. From this perspective, it is the number of carbon atoms per area within the CNT film, absorbing or attenuating the EUV light, which should be controlled. For a free-standing SWCNT film with an EUV transmission of $96 \%$ the amount of carbon atoms was measured to be around $2 \cdot 10^{16}( \pm 2 \%)$ at $/ \mathrm{cm}^{2}$ using ERD technique ${ }^{23}$, which is equivalent to a carbon layer of $3 \mathrm{~nm}$ thick (assuming the density of $1.33 \mathrm{~g} / \mathrm{cm}^{324}$ ).

The degree of CNT bundling, which typically occurs during the CNT synthesis and membrane formation, plays an important role in tuning the CNT film properties ${ }^{25}$. Our results indicate that for comparable EUV transmission levels of the CNT membrane, larger bundle diameters improve the film's emissivity. A similar trend is visible for diameter variations of individual CNTs. When the CNT diameter is increased from $\sim 2 \mathrm{~nm}$ (SWCNT) to $\sim 8 \mathrm{~nm}$ (MWCNT), the emissivity of the CNT film is increased $\sim 2.5$ times for comparable EUV transmission values. However, emissivity is not the only relevant parameter. When the size of the CNT fine structure approaches the wavelength, EUV scattering starts to play a role. This was investigated in details at PTB. Low scattering was measured from the low-density SWCNT film, which was at a level comparable to a SiN reference membrane. Intense scattering was measured from some samples of the high-density MWCNT films ${ }^{26}$.

These results suggest a direction for tuning the CNT membrane properties to achieve the optimal configuration. There is always a tradeoff between a number of desired specifications, like EUV transmission, scattering, emissivity, membrane strength and gap size distribution.

\subsection{Summary of the CNT membrane properties}

The free-standing CNT membrane demonstrates a combination of desirable properties with respect to the EUV pellicle solution (see table 2). Besides high EUV transmission, uniformity and mechanical stability of the CNT membrane, the ability of the CNT membrane to reflect light was also assessed. Since the EUV light reflected at the pellicle will add to the flare level, it has to be minimized with the developmental goal of less than $0.04 \%{ }^{16}$. CNT membranes showed no detectable EUV reflectance above the noise level of $0.001 \%$ as measured at PTB $^{26}$. This was explained by the roughness and irregularity of the CNT structures comprising the membrane as opposed to the smooth SiN films ${ }^{26}$. Another favourable CNT membrane property for the pellicle application is its ability to transmit light at $193 \mathrm{~nm}$, allowing through-pellicle DUV mask inspection. Transmission of the CNT membrane vs wavelength in the range of 190-250 nm is shown in Figure 7.

Table 2: Properties of the CNT membrane.

\begin{tabular}{|l|l|l|}
\hline Membrane property & Desired $^{16}$ & Achieved with CNT membrane \\
\hline EUV Transmission & $>90 \%$ & $>96 \%$ \\
\hline EUV Transmission uniformity & $0.4 \%$ half range & $0.4 \%$ half range \\
\hline EUV reflectivity & $0.04 \%$ & noise level: $0.001 \%{ }^{26}$ \\
\hline $\begin{array}{l}\text { Mechanical stability (film deflection } \\
\text { at 2Pa dP) }\end{array}$ & $\begin{array}{l}<0.5 \mathrm{~mm} \\
* \text { for } 110 \times 140 \mathrm{~mm}^{2}\end{array}$ & $\begin{array}{l}\sim 0.08 \mathrm{~mm} \\
* \text { measured for } 10 \times 10 \mathrm{~mm}^{2}\end{array}$ \\
\hline $\begin{array}{l}\text { Through-pellicle DUV mask } \\
\text { inspection }\end{array}$ & possible & $\begin{array}{l}\text { possible } \\
\sim 83 \% \text { transmission @ } 193 \mathrm{~nm}\end{array}$ \\
\hline EUV scattering & $<0.25 \%$ & depends on CNT type \\
\hline
\end{tabular}




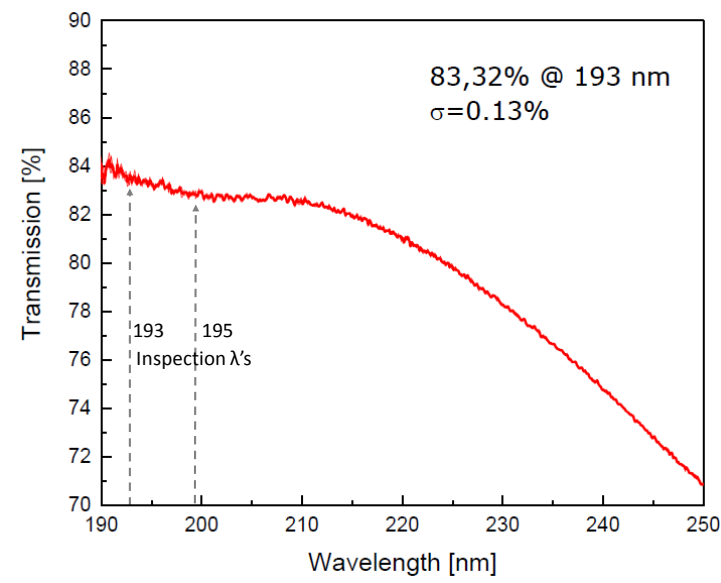

Figure 7: Transmission of the CNT membrane vs wavelength showing the capability of through-pellicle DUV mask inspection. The EUV transmission of this film was measured to be $96 \%$. The measurements were performed at RWTH Aachen University.

\section{TOWARDS A FULL-SIZE SOLUTION}

\subsection{Scaling the CNT membrane to the full pellicle size}

When exploring potential material solutions for the EUV pellicle application, the ability to fabricate the pellicle at the required size in order to fully cover the mask pattern area is essential. The advantage of the CNT material is its scalability and good handling strength. Figure 8 demonstrates the free-standing CNT film with the inner film area of 120 $\mathrm{mm} \times 150 \mathrm{~mm}$, having an aspect ratio of length/thickness of $>10^{6}$. The EUV transmission of this film was measured to be $96 \%$.

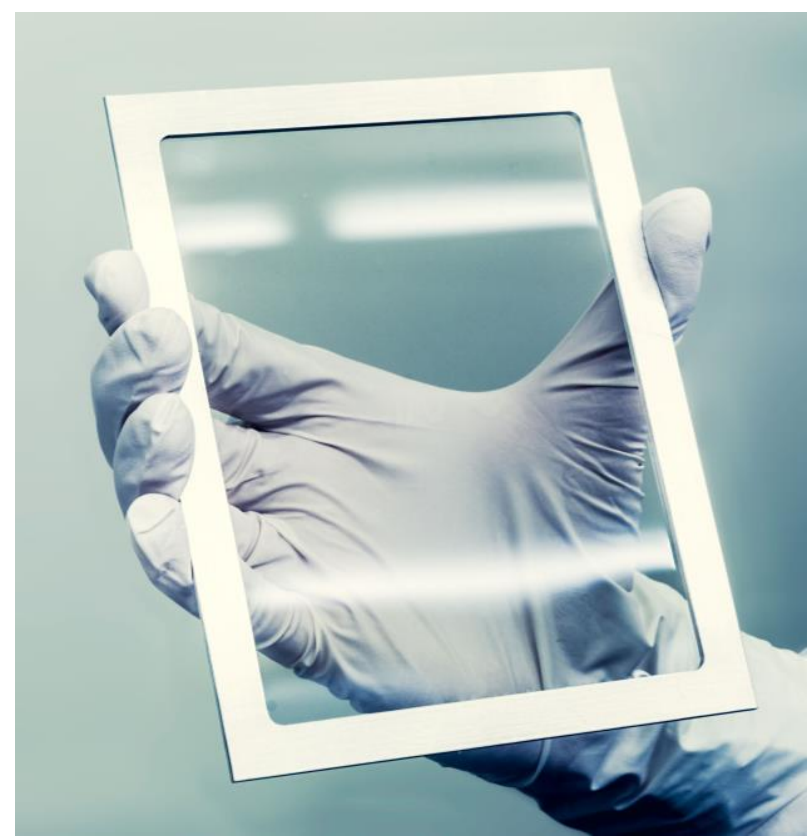

Figure 8: Free-standing CNT membrane at full pellicle size.

\subsection{Chemical stability in the current scanner environment}

The only known failure for the CNT core solution is its instability in the current scanner environment of EUV $+\mathrm{H}_{2}$. Hydrogen radicals $\left(\mathrm{H}^{*}\right)$ are introduced to the EUV scanner to clean the system from carbon contamination. It is known that harsh H-plasma and higher reaction temperatures irreversibly etch and eventually completely remove CNTs with the 
formation of hydrocarbon molecules ${ }^{27}$. It was explored that $\mathrm{H}$-etching at high temperatures is likely to start at defect sites of the CNTs (or strained curved sites) and further propagates along the tube length ${ }^{27}$. Figure 9 (a-b) demonstrates that after exposing the CNT film to $\mathrm{H}^{*}$ for 110 hours at room temperature most of the CNT material is removed ${ }^{28}$. This test was done on a random SWCNT network, however a similar result was also observed for MWCNT films. The instability of CNTs in the environment of $\mathrm{H}^{*}$ dictates the need for coating the CNT core material. A coating is already required for the existing thin film pellicle solutions to, for example, enhance emissivity ${ }^{5}$.

Figure 9 (c-d) shows a CNT film on a silicon substrate coated with a thin molybdenum (Mo) layer (4 $\mathrm{nm}$ ) before and after exposure to $\mathrm{H}^{*}$ environment at room temperature for 110 hours. No significant CNT density decrease is seen. In addition to the visual examination of the CNT film density in SEM, we measured the amount of carbon atoms present on the surface for both uncoated and Mo-coated CNT films before and after exposure to $\mathrm{H}^{*}$ at room temperature by means of elastic recoil detection (ERD) analysis. Figure 9 (e) displays ERD results for the areal density of carbon, showing that the amount of carbon drops significantly for the uncoated CNT film after exposure to $\mathrm{H}^{*}$ but stays on the same level for Mo-coated CNT film. Furthermore, the structural modification generated on the surface of the CNT films was assessed using Raman spectroscopy. The ratio of intensities between the 'D band' at around $1300 \mathrm{~cm}^{-1}$ and the ' $\mathrm{G}$ band' at 1590 $\mathrm{cm}^{-1}\left(\mathrm{I}_{\mathrm{D}} / \mathrm{I}_{\mathrm{G}}\right)$ was compared for Mo-coated CNT film before and after it was exposed to $\mathrm{H}^{*}$ at room temperature. Raman results indicate that $\mathrm{I}_{\mathrm{D}} / \mathrm{I}_{\mathrm{G}}$ ratio of this coated CNT film was not significantly altered after exposure. Moreover, TEM investigation showed a uniform coating of SWCNTs and bundles with Mo (inset Figure 9 (f)). These results suggest that Mo coating provides protection of the CNT film from $\mathrm{H}^{*}$ at room temperature, however coating thickness could still be optimized. Note that in these tests the exposure to $\mathrm{H}^{*}$ occurs at room temperature. In the real scanner environment, elevated temperatures are expected to make the conditions harsher for the stability of the pellicle membrane. CNT membrane coated with $4 \mathrm{~nm}$ Mo on both sides demonstrated 92\% EUV transmission.

(a)

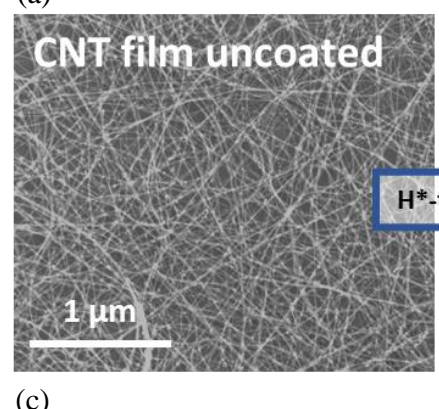

(b)

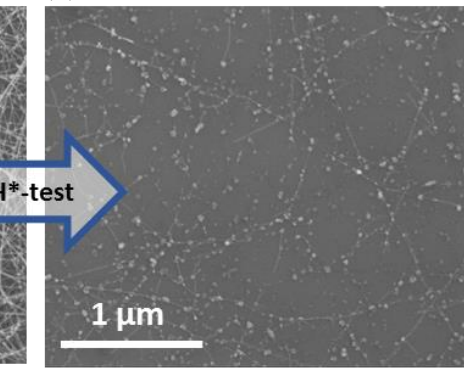

(d) (f)
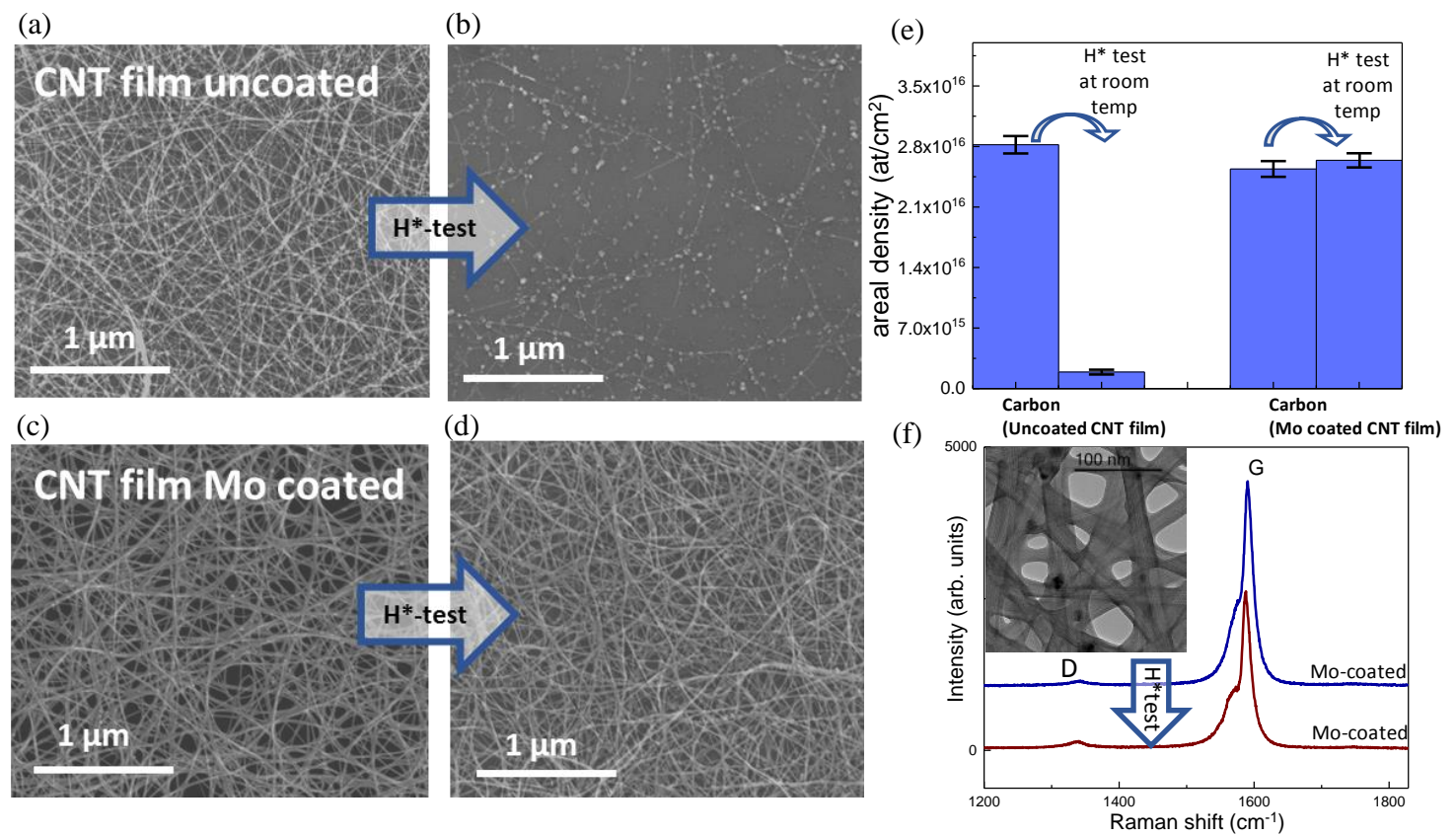

Figure 9: SEM of the CNT film on a silicon substrate (a) before and (b) after the exposure to the environment of hydrogen radicals at room temperature. SEM of Mo-coated $(4 \mathrm{~nm})$ CNT film on a silicon substrate (a) before and (b) after the exposure to the environment of hydrogen radicals at room temperature. (e) ERD of uncoated and Mo-coated CNT film before and after exposure to hydrogen radicals environment. (f) Raman spectra of Mo-coated CNT film before and after exposure to the hydrogen radical environment. Inset: Mo-coated SWCNTs and bundles.

\subsection{Coating path to a complete CNT EUV pellicle}

For the CNT core material to be compatible with the current scanner environment, the carbon structures must be coated to prevent $\mathrm{H}^{*}$ etching. Some requirements for pellicle protective layers are shared with conventional EUV pellicle 
solutions: optical properties, i.e. the $\mathrm{n}$ and $\mathrm{k}$ values must be such that the full membrane solution meets the target for high EUV transmission, low reflection and scattering, and thermal properties, i.e. compatibility with greater than $250 \mathrm{~W}$ exposure in the scanner.

When other targets are met, EUV scattering is emerging as a challenge for the pellicle application. The pellicle is in the optical path for imaging. If EUV exposure light is scattered outside of the numerical aperture (NA) of the optics, it is transmission loss. If the exposure light is scattered into the collection cone, the effect is more like flare and must be minimized. A systematic study of the desirable properties with respect to both EUV transmission and scattering was undertaken to understand both the impact of coatings and the effect of the CNTs within the core material. To estimate the impact of a coating on EUV scattering and transmission, rigorous calculations of far field optical parameters are needed. This is essentially Mie scattering and the approximation of infinitely long cylindrical structures can be used to represent the CNT network ${ }^{29}$. For this approach, an open source MATLAB script is available and was used for the calculations here ${ }^{30}$. For the pellicle application, several factors must be considered, i.e.

- Extinction of light contributes to transmission loss

- Scattering of light outside the cone of the projection lens (NA) is transmission loss

- Scattering of light within the cone of the projection lens (NA) is not only transmission loss, but can affect the imaging

- $\quad$ Transmission loss $=1-($ extinction + total scattering $)$

Using the approximation of simple cylindrical structures, this calculation provides both the transmission loss as well as the scatter within the numerical aperture of the scanner (see Figure 10 (a)). The impact of bundling and deposited coatings on these results is captured in the model as well. A schematic of this is shown in Figure 10 (b). The scattering and extinction of a bundle made of a number $(\mathrm{N})$ of CNTs is similar to that of a single cylinder having the same volume. In other words, the same cross-sectional area in this approximation can be used. If the diameter of a single CNT is d, then the equivalent bundle of N CNTs has a diameter of $(\sqrt{ } \mathrm{N} \cdot \mathrm{d})$. For the coating, a uniform thickness is assumed, and for the optical constant of the coated CNT a weighted average of the individual optical constants can be used (Figure 10 (b)). Since there are many approximations, the primary goal of the simulations is to identify trends for the optimized CNT pellicle membranes.

(a)

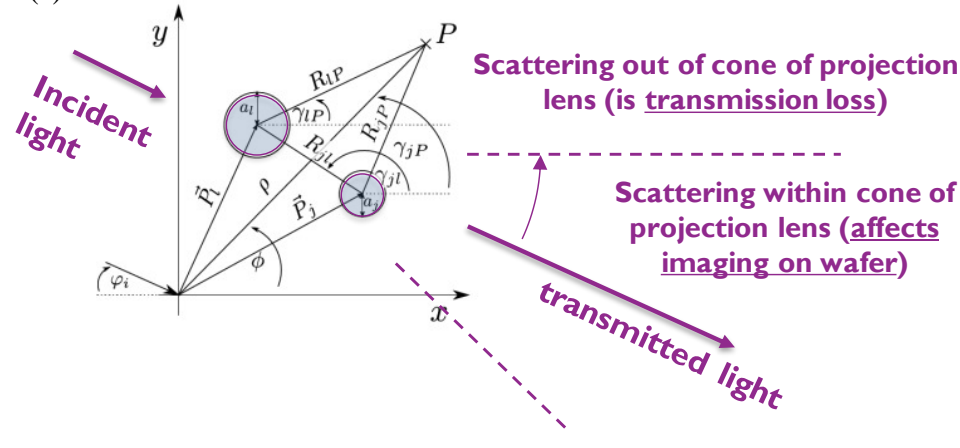

(b)

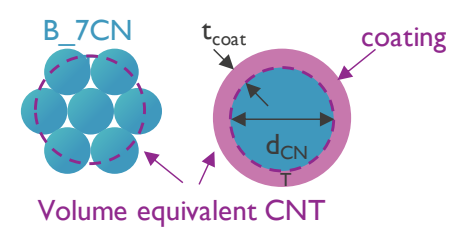

Weight factor for CNT: $\mathrm{W} 1=\pi\left(\mathrm{d}_{\mathrm{CNT}} / 2\right)^{2}$

Weight factor for coat: $\mathrm{W} 2=\pi\left(\mathrm{d}_{\mathrm{CNT}} / 2+\mathrm{t}_{\mathrm{coat}}\right)^{2}-\pi\left(\mathrm{d}_{\mathrm{CNT}} / 2\right)^{2}$

Optical constant for coated CNT : $\left(\mathrm{W} 1 \mathrm{n}_{\mathrm{CNT}}+\mathrm{W} 2 \mathrm{n}_{\mathrm{coat}}\right) /(\mathrm{W} 1+\mathrm{W} 2)$

Figure 10: (a) An illustration of the principles for calculating optical properties of the CNT pellicle based on Schaeffer $(2012)^{30}$. (b) A schematic illustration of the approximations used in calculating coated CNTs and bundles.

EUV scattering is expected to depend heavily on the optical constant of the coating material. Therefore for a given CNT geometry (for example, CNT diameter $=5 \mathrm{~nm}$; CNT-to-CNT pitch $=55 \mathrm{~nm}$ ), the total scatter into the $0.33 \mathrm{NA}$ can be calculated as a function of optical constants $(\mathrm{n}, \mathrm{k})$ and thickness of a coating. Fixing the coating thickness at $4 \mathrm{~nm}$, the results are shown in Figure 11 (a). It is visible that Mo coating film is located on the contour plot and shows a scatter of roughly $1 \%$. This value is too high with respect to imaging requirements. Consequently, a material with (n,k) closer to $(1,0)$ is preferred. 

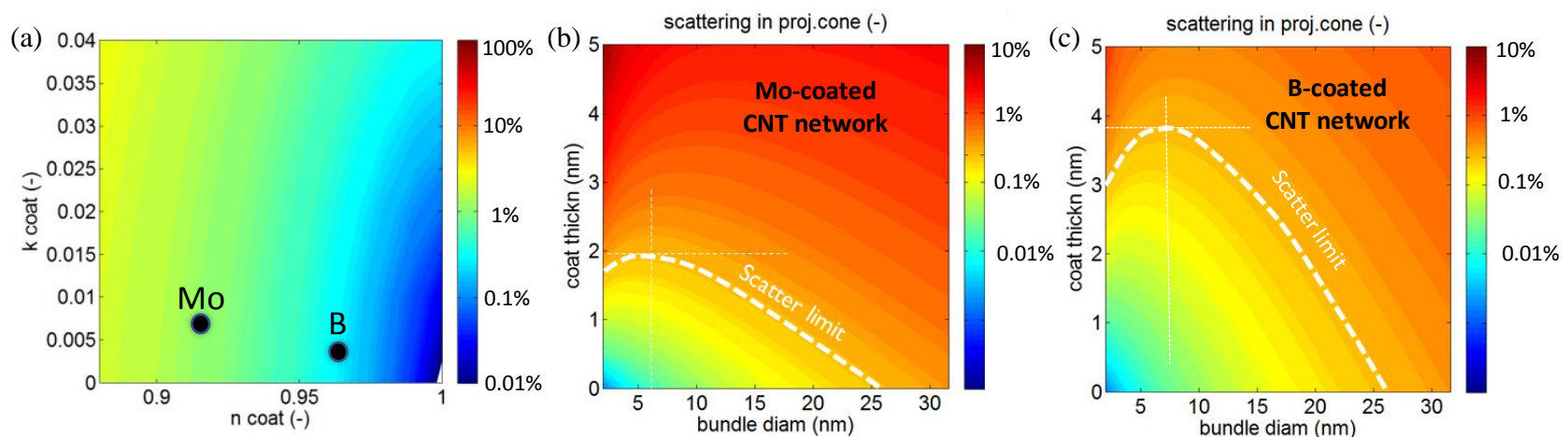

Figure 11: (a) A contour plot of the calculated total scatter into NA as a function of (n,k) for a defined CNT geometry and coating thickness of $4 \mathrm{~nm}$. Mo $(\mathrm{n}=0.924, \mathrm{k}=0.006)$ and $\mathrm{B}(\mathrm{n}=0.968, \mathrm{k}=0.004)$ are shown as two examples. Scattering simulations for (b) Mo-coated CNT network and (c) B-coated CNT network, where bundle diameter and coating layer thickness are varied.

For the selected coating examples, we estimate the impact of both the CNT bundling within the membrane and the coating thickness on the EUV scatter. The number of CNTs is fixed over a unit area of the pellicle, but different levels of bundling are assumed. Since the number of CNTs is constant, the bundling increases the pitch between bundles. For example, if a bundle contains N CNTs, the pitch of this bundle will be N times the pitch of the individual CNT. The degree of bundling is characterized by a volume-equivalent bundle diameter, and the impact of the coating thickness is investigated using the optical constants $(\mathrm{n}, \mathrm{k})$. Figure 11 (b) shows the initial scattering model into the NA cone for a CNT film coated with Mo. The diameter of individual CNTs within the film in this case is $2 \mathrm{~nm}$ and CNT-to-CNT pitch is $6 \mathrm{~nm}$. There is an optimal bundling diameter that minimizes scatter. In the plot, a suggested scatter target of $0.25 \%$ is shown. If this is required, the coating thickness should be $2 \mathrm{~nm}$ or less with bundle diameter as close as possible to $6 \mathrm{~nm}$. If the scatter is controlled, the EUV transmission is expected to exceed the target of $90 \%$.

In a second example, the same CNT network is coated with a material that is halfway between Mo $(n, k)$ and $(1,0)$, i.e. boron (B). All other parameters remain the same. The result of scattering is shown in Figure 11 (c). The scatter plot indicates that a much thicker coating (almost $4 \mathrm{~nm}$ ) is acceptable, keeping the scattering within the suggested limit. The optimum bundling here is close to a $7 \mathrm{~nm}$ bundle diameter. Once again, transmission loss is not an issue if scattering is controlled.

Besides the choice of material itself, the coating process must be compatible with the free-standing CNT structure and result in uniform and conformal coating all around the individual CNTs and their bundles. A broad range of thin film deposition techniques exists: physical vapor deposition (PVD) (including sputtering, electron-beam evaporation, pulsed laser deposition), chemical vapor deposition (CVD) and atomic layer deposition (ALD), which can be used for coating the CNTs. However, not all processes are compatible with the free-standing CNT membrane, especially when it is comprised of SWCNTs. For example, plasma-based processes introduce structural damage to the CNTs which typically leads to breaking of the SWCNT membrane. Figure 12 (a) demonstrates an example showing compatibility of different thin film deposition processes with a highly transparent SWCNT membrane (EUV transmission >96\%) based on the analysis of $\mathrm{I}_{\mathrm{D}} / \mathrm{I}_{\mathrm{G}}$ ratio from Raman spectroscopy. The lower $\mathrm{I}_{\mathrm{D}} / \mathrm{I}_{\mathrm{G}}$ ratio points at fewer defects and therefore higher structural quality of the CNTs. Electron-beam evaporation and certain ALD processes proved to be compatible with the SWCNT membrane, providing the possibility for a multi-layer coating. Figure 12 (b) shows example TEM images of SWCNTs coated by different methods, demonstrating the challenge to achieve uniform and conformal coating all around the SWCNTs and their bundles with certain techniques, like ALD $^{31}$. Careful selection of the coating materials and coating processes or their combinations is vital to protect the core CNT material to make it a viable EUV pellicle solution for the current scanner environment. 

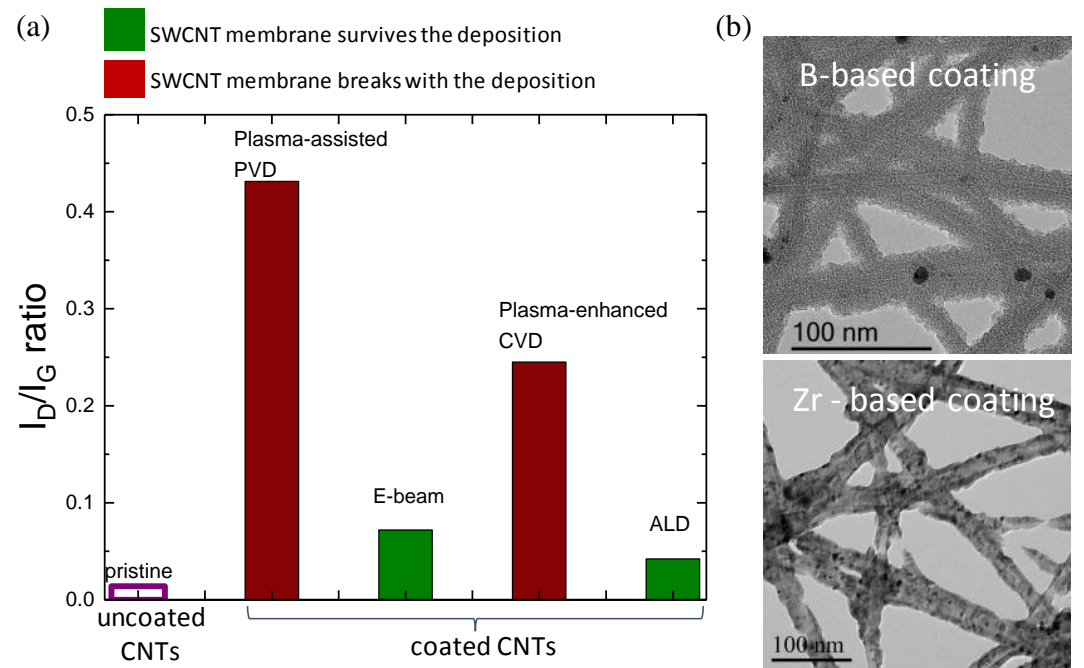

Figure 12. (a) Compatibility of different deposition techniques to coat a highly transparent SWCNT membrane (EUV transmission $>96 \%$ ) based on the analysis of the ratio of $\mathrm{D}$ to $\mathrm{G}$ peak intensities $\left(\mathrm{I}_{\mathrm{D}} / \mathrm{I}_{\mathrm{G}}\right)$ using Raman spectroscopy. (b) TEM images of SWCNTs coated with B-based material by PVD and Zr-based material by ALD.

\section{SUMMARY}

We propose an alternative pellicle membrane made of CNTs as a core pellicle solution to meet high volume manufacturing requirements of beyond $250 \mathrm{~W}$ EUV exposure. We explore the properties of the free-standing CNT film, demonstrating its outstanding EUV transmission above $96 \%$ and acceptable transmission uniformity level of $0.4 \%$ half range, good mechanical strength at full pellicle size due to permeability of the CNT film and thermal stability with no change when exposed up to $1500 \mathrm{~W}$ source power equivalent without hydrogen radicals. The ability of such porous membranes to stop particles is modeled based on SEM analysis and the results indicate that the probability of small particles $(30-60 \mathrm{~nm})$ to pass through the membrane is only a few percent even using worst-case assumptions, and for larger particles it decreases exponentially. Experimental verification of these results is in progress. The direction towards tuning the thermo-optical properties of the membrane via altering the characteristics of individual CNT components is identified based on the experimental results. We show that the major obstacle for the CNT core material to become a viable pellicle solution is its instability in the environment of hydrogen radicals/ions of the current scanner. The path towards protective coating of the CNT structures is explored. The challenges of coating CNTs related to the right choice of the coating material and the deposition process are identified.

\section{ACKNOWLEDGEMENTS}

We would like to thank CNT suppliers: Canatu Oy and Lintec Ltd. We acknowledge the contribution of Albert G. Nasibulin and Vsevolod Iakovlev (Skolkovo Insitute of Technology) at the start of the free-standing CNT membrane research. We thank Frank Scholze and Christian Laubis (PTB) for scattering measurements; Sascha Brose and Serhiy Danylyuk (RWTH) for additional optical measurements; Chami Perera (EUV Tech) for EUV transmission uniformity measurements. We appreciate the assistance of Elie Schapmans, Kim Baumans, Sander Van Gompel, Kenny Leyssens, Jeroen van Swigchem, Joren van Kempen, Thomas Witters, Rudy Verheyen, Nicolò Pinna, Thomas Vandewal, Thibaut Maurice with thin film deposition and coating development. We also acknowledge Xiangyu Wu, Ken Verguts, Yao Teng Chuang, Marcel Lux, Thomas Nuytten for Raman analysis. We thank Fred Loosen for the photos. This project has received partial funding from the Electronic Component Systems for European Leadership Joint Undertaking under grant agreement No 662338. This Joint Undertaking receives support from the European Union's Horizon 2020 research and innovation programme and Netherlands, France, Belgium, Germany, Czech Republic, Austria, Hungary and Israel. 


\section{REFERENCES}

[1] Turkot, B., Carson, S. L., Lio, A., Liang, T., Phillips, M., McCool, B., Stenehjem, E., Crimmins, T., Zhang, G. and Sivakumar, S., "EUV progress toward HVM readiness,” Proc. SPIE 9776, EUV Lithogr. VII 977602 (2016).

[2] Smith, D., “ASML NXE Pellicle progress update,” EUV Mask Pellicle TWG, San Jose, CA (2016).

[3] Goldfarb D. L., "Fabrication of a full-size EUV pellicle based on silicon nitride,” Proc. SPIE 9635, Photomask Technol. 96350A (2015).

[4] Kim, M. J., Jeon, H. C., Chalykh, R., Kim, E., Na, J., Kim, B.-G., Kim, H., Jeon, C., Kim, S.-G., Shin, D.-W., Kim, T., Kim, S., Lee, J. H. and Yoo, J.-B., "Study of nanometer-thick graphite film for high-power EUVL pellicle,” Proc. SPIE 9776, EUV Lithogr. VII $97761 Z$ (2016).

[5] Zwol, P. J. van, Nasalevich, M., Kurganova, E., Notenboom, A., Voorthuijzen, P., Vles, D., Péter, M., Symens, W., Giesbers, A. J. M. (Jos), Klootwijk, J., van de Kruijs, R. W. E. and van der Zande, W., "Pellicle films supporting the ramp to HVM with EUV,” Proc. SPIE Int. Conf. on EUV Lithogr. 10451-22 (2017).

[6] Pollentier, I., Vanpaemel, J., Lee, J. U., Adelmann, C., Zahedmanesh, H., Huyghebaert, C. and Gallagher, E. E., "EUV lithography imaging using novel pellicle membranes," Proc. Vol. 9776, Extrem. Ultrav. Lithogr. VII; 977620 (2016).

[7] Wood, J., "The top ten advances in materials science," Mater. Today 11(1-2), 40-45 (2008).

[8] Chang, C.-C., Hsu, I.-K., Aykol, M., Hung, W.-H., Chen, C.-C. and Cronin, S. B., “A New Lower Limit for the Ultimate Breaking Strain of Carbon Nanotubes," ACS Nano 4(9), 5095-5100 (2010).

[9] Kim, P., Shi, L., Majumdar, A. and McEuen, P. L., “Thermal Transport Measurements of Individual Multiwalled Nanotube,” Phys. Rev. Lett. 87(21), 215502-1-4 (2001).

[10] De Volder, M. F., Tawfick, S. H., Baughman, R. H. and Hart, A. J., "Carbon nanotubes: present and future commercial applications," Science 339(6119), 535-539 (2013).

[11] Wang, X., Li, Q., Xie, J., Jin, Z., Wang, J., Li, Y., Jiang, K. and Fan, S., "Fabrication of Ultralong and Electrically Uniform Single-Walled Carbon Nanotubes on Clean Substrates,” Nano Lett. 9(9), 3137-3141 (2009).

[12] Nasibulin, A. G., Kaskela, A. O., Mustonen, K., Anisimov, A. S., Ruiz, V., Kivistö, S., Rackauskas, S., Timmermans, M. Y., Pudas, M., Aitchison, B., Kauppinen, M., Brown, D. P. and Okhotnikov, O.G., Kauppinen, E. I., "Multifunctional free-standing single-walled carbon nanotube films,” ACS Nano 5(4), 3214-3221 (2011).

[13] Hennrich, F., Lebedkin, S., Malik, S., Tracy, J., Barczewski, M., Rösnera, H. and Kappesab, M., "Preparation, characterization and applications of free-standing single walled carbon nanotube thin films," Phys. Chem. Chem. Phys. 4, 2273-2277 (2002).

[14] Shi, Z., Chen, X., Wang, X., Zhang, T. and Jin, J., "Fabrication of superstrong ultrathin free-standing single- 
walled carbon nanotube films via a wet process," Adv. Funct. Mater. 21, 4358-4363 (2011).

[15] Zhang, M., Fang, S., Zakhidov, A. A., Lee, S. B., Aliev, A. E., Williams, C. D., Atkinson, K. R. and Baughman, R. H., "Strong, Transparent, Multifunctional, Carbon Nanotube Sheets," Science 309(5738), 1215-1219 (2005).

[16] Brouns, D., "Pellicle HVM specifications," Pellicle TWG, San Jose, CA (2016).

[17] Pollentier, I., Lee, J. U., Timmermans, M. Y., Adelmann, C., Zahedmanesh, H., Huyghebaert, C. and Gallagher, E. E., "Novel membrane solutions for the EUV pellicle: better or not?," Proc. SPIE 10143, EUV Lithogr. VIII, 101430L (2017).

[18] van Es, R., "EUV lithography industrialization progress," Intern. Conf. on EUV Lithogr, 10450-2 (2017).

[19] Zhang, G., Jiang, S., Yao, W. and Liu, C., "Enhancement of Natural Convection by Carbon Nanotube Films Covered Microchannel-Surface for Passive Electronic Cooling Devices,” ACS Appl. Mater. Interfaces 8, 3120231211 (2016).

[20] Jiang, S., Liu, C. and Fan, S., "Efficient Natural-Convective Heat Transfer Properties of Carbon Nanotube Sheets and Their Roles on the Thermal Dissipation,” ACS Appl. Mater. Interfaces 6, 3075-3080 (2014).

[21] Thostenson, E. T., Li, C. and Chou, T., "Nanocomposites in context," Compos. Sci. Technol. 65, 491-516 (2005).

[22] Laubis, C., “Membrane sample EUV characterization,” IEUVI Pellicle TWG Meet. SPIE, San Jose (2016).

[23] Meersschaut, J. and Vandervorst, W., "Nuclear Instruments and Methods in Physics Research B Highthroughput ion beam analysis at imec,” Nucl. Inst. Methods Phys. Res. B 406, 25-29 (2017).

[24] Collins, P. G. and Avouris, P., "Nanotubes for electronics,” Sci. Am. 283(6), 62-69 (2000).

[25] Kaskela, A., Nasibulin, A.G., Timmermans, M.Y., Aitchison, B., Papadimitratos, A., Tian, Y., Zhu, Z., Jiang, H., Brown, D. P., Zakhidov, A. and Kauppinen, E. I., “Aerosol-synthesized single-walled carbon nanotube networks with tunable conductivity and transparency by dry transfer technique," Nano Lett. 10, 4349-4355 (2010).

[26] Scholze, F., Laubis, C., Krumrey, M., Timmermans, M. Y., Pollentier, I. and Gallagher, E. E., "EUV optical characterization of alternative membrane materials for EUV pellicles," Proc. SPIE Int. Conf. on EUV Lithogr.

10451-25 (2017).

[27] Zhang, G., Qi, P., Wang, X., Lu, Y., Mann, D., Li, X. and Dai, H., "Hydrogenation and hydro-carbonation and etching of single-walled carbon nanotubes," J. Am. Chem. Soc. 128(18) (2006).

[28] Pollentier, I. and et al., "Optical testing of EUV pellicle materials," Int. EUVL Symp. Maastricht (2015).

[29] Lee, S., "Dependent scattering of an obliquely incident plane wave by a collection of parallel cylinders," J. Appl. Phys. 68, 4952 (1990). 
[30] Jan, S., "Solve the scattering of electromagnetic radiation by a sphere or multiple cylinders". MatScat. version 1.4 (2012).

[31] Farmer, D. B. and Gordon, R. G., "Atomic layer deposition on suspended single-walled carbon nanotubes via gas-phase noncovalent functionalization," Nano Lett. 6(4), 699-703 (2006). 